\title{
Electronic-enthalpy functional for finite systems under pressure
}

\author{
Matteo Cococcioni, ${ }^{1}$ Francesco Mauri, ${ }^{2}$ Gerbrand Ceder,${ }^{1}$ and Nicola Marzari ${ }^{1}$ \\ ${ }^{1}$ Department of Materials Science and Engineering, and Institute for Soldier Nanotechnologies, \\ Massachusetts Institute of Technology, 77 Massachusetts Avenue, Cambridge, MA 02139, USA \\ ${ }^{2}$ Laboratoire de Minéralogie-Cristallographie de Paris, \\ Université Pierre et Marie Curie, 4 Place Jussieu, 75252, Paris, Cedex 05, France
}

(Dated: November 9, 2018)

\begin{abstract}
We introduce the notion of electronic enthalpy for first-principles structural and dynamical calculations of finite systems under pressure. An external pressure field is allowed to act directly on the electronic structure of the system studied via the ground-state minimization of the functional $E+P V_{q}$, where $V_{q}$ is the quantum volume enclosed by a charge isosurface. The Hellmann-Feynman theorem applies, and assures that the ionic equations of motion follow an isoenthalpic dynamics. No pressurizing medium is explicitly required, while coatings of environmental ions or ligands can be introduced if chemically relevant. We apply this novel approach to the study of group-IV nanoparticles during a shock wave, highlighting the significant differences in the plastic or elastic response of the diamond cage under load, and their potential use as novel nanostructured impact-absorbing materials.
\end{abstract}

The study of nanoparticles under pressure is rapidly acquiring great scientific and technological interest, since it allows to explore structural arrangements and phase transitions under different kinetic and thermodynamic conditions than those of bulk solids. While experiments on the compression of nanoparticles have been performed for more than a decade [1, 2, 3, 4, 5], and encompass broad classes of materials [6, 7], computational studies have been hampered by the conceptual difficulty arising in applying classical or electronic-structure methods to the study of finite systems under pressure. Extended systems can be studied using variable-cell isoenthalpic dynamics 8, 9, 10, 11]; however, these methods do not carry over to the case of finite systems, unless the environment (i.e. the pressurizing medium) is introduced explicitly in the calculations [12, 13, 14]. A full quantummechanical treatment becomes then too costly in all but the smallest cases, due to the large number of atoms or molecules that are needed to reproduce the pressurizing environment; in addition, equilibration of the system at a given pressure requires extensive simulations averaging over many collision events. To solve the first of these problems, a mixed quantum-mechanics/molecularmechanics approach has been proposed 12, 13] where the quantum fragment is compressed by a pressurizing medium described by classical force fields.

In this Letter we present a novel and general approach to this problem, and show how pressure can be applied to a finite system without the need to introduce a pressurizing medium. We introduce here, as thermodynamic functional describing a finite system under pressure, the electronic enthalpy

$$
H=E+P V_{q},
$$

where $E$ is the internal energy of the system, $P$ is the desired pressure and $V_{q}$ is the "quantum volume" occupied by the electronic charge density. This quantity is well defined once the threshold for a density isosurface is chosen, and can be straightforwardly computed as

$$
V_{q}=\int d \mathbf{r} \vartheta(\rho(\mathbf{r})-\alpha)
$$

where $\rho(\mathbf{r})$ is the electronic density and $\vartheta$ is a step function at the threshold value $\alpha$. For computational convenience we smooth the $\vartheta$ to $\tilde{\vartheta}$, defined as the integral of a normalized Gaussian of width $\sigma=\alpha / 3$ [15].

In density-functional theory, the total potential acting on the electrons will be given by the functional derivative of the electronic enthalpy in (10) with respect to the charge density $\rho(\mathbf{r})$. This results in an additional contribution deriving from the term $P V_{q}$ which is simply

$$
\Phi_{V}(\mathbf{r})=\left.P \frac{\delta V_{q}}{\delta \rho}\right|_{\rho=\rho(\mathbf{r})}=\frac{P}{\sigma \sqrt{2 \pi}} e^{-(\rho(\mathbf{r})-\alpha)^{2} / 2 \sigma^{2}} .
$$

In the self-consistent solution of the Kohn-Sham equations the $\Phi_{V}$ potential drives the evolution of the electronic ground-state to the minimum of the electronic enthalpy (11). The Hellmann-Feynman theorem applies, and thus the ionic forces take implicitly into account the contribution of the external load, transferring the effects of the compression directly to the ionic relaxation and dynamics. A Lagrangian formulation (e.g. for firstprinciples Car-Parrinello molecular dynamics [16]) also follows directly.

The introduction of an electronic-enthalpy functional has several conceptual and practical advantages: 1) The pressure field acts directly on the electrons; thus, the compressibility of the system is properly dominated by electrostatic and Pauli-principle effects. Also, if pressure were transferred directly to the ionic nuclei by an external classical force field, electrons could ultimately be squeezed out of the system. 2) Isoenthalpic relaxations and dynamics do not require extensive equilibration with a pressurizing medium; a medium can always be introduced as an environmental coating, if chemically rele- 


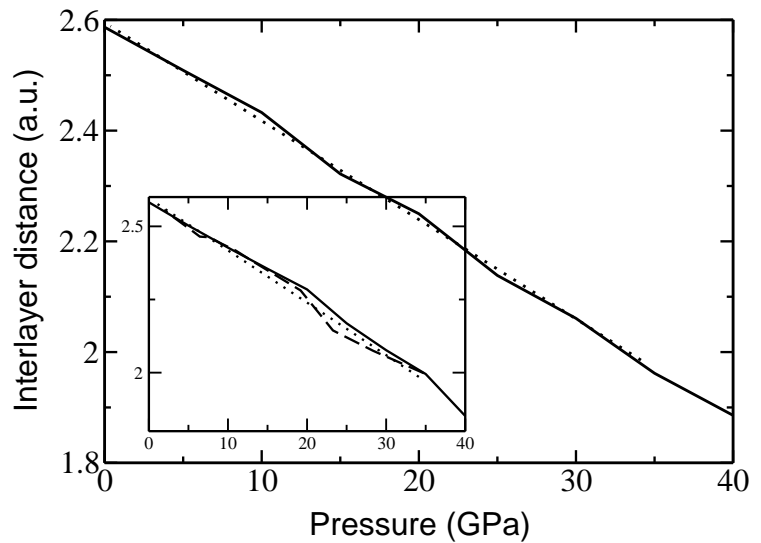

FIG. 1: The dependence of the innermost interlayer distance on pressure for a 16-layer silicon slab relaxed using the electronic-enthalpy functional (solid curve); results from the Murnaghan equation of state for a 16-layer bulk supercell (dotted) are shown for comparison. The inset shows the results obtained for a 8-layer slab (solid), a 8-layer supercell (dotted), and a 8-layer slab compressed via the explicit introduction of argon as a pressurizing medium (dashed).

vant. 3) In the thermodynamic limit, the classical macroscopic volume and enthalpy are recovered. 4) Implementation in electronic-structure codes is straightforward, and can be easily extended to other wavefunction-based approaches such as Hartree-Fock or variational quantum Monte Carlo. (The results presented here have all been obtained using first-principles Car-Parrinello molecular dynamics [16], as implemented in the public domain $\nu$-ESPRESSO package [17].)

Other developments and applications stemming from this formulation can be envisioned. A surface tension term $H \rightarrow H+\gamma S$ can be introduced, where the surface area is obtained by finite differences via

$S=\int d \mathbf{r}\left[\tilde{\vartheta}\left(\rho(\mathbf{r})-\left(\alpha-\frac{\Delta}{2}\right)\right)-\tilde{\vartheta}\left(\rho(\mathbf{r})-\left(\alpha+\frac{\Delta}{2}\right)\right)\right] \frac{|\vec{\nabla} \rho(\mathbf{r})|}{\Delta}$

and $\Delta$ determines the width of the discretization. This latter contribution to the energy functional could be used to characterize surface tension in metallic nanoparticles, confinement effects in electron bubbles (e.g., for electrons in superfluid helium 18]) or cavitation effects in solvation models [19. Finally, we stress that although classical definitions of volume can be introduced in atomistic simulations [20, 21], a natural measure based on the electron density can only emerge in a quantum-mechanical formalism. A validation of the present approach can be provided not only in the thermodynamical limit (where the quantum volume $\mathrm{V}_{q}$ becomes indistinguishable from the classical limit), but also for finite, small systems. For this purpose we studied the effect of pressure on thin silicon slabs. This reference system allows for a direct comparison with bulk results without introducing additional surface tension effects. We plot in Fig. 1 the average distance between the innermost layers in two $\mathrm{Si}(001)$ slabs of 8 and 16 layers, as a function of the pressure parameter $\mathrm{P}$ entering our functional (11). A remarkable agreement is found with the results obtained when calculating the physical pressure 22] in a bulk silicon system having the same geometry and compressed along the [001] direction. For the 8-layer slab a full quantum-mechanical calculation taking into account explicitly argon as a pressurizing medium has been performed, delivering an even closer agreement (at least up to $20 \mathrm{GPa}$ ) to our electronicenthalpy results. This agreement clearly demonstrates that the minimization of the quantum enthalpy correctly accounts for the effects of pressure; in addition, agreement is reached rapidly with respect to system size 23].

Our case studies are group-IV nanoparticles $\mathrm{M}_{35} \mathrm{H}_{36}$ during a shock wave (M corresponds to $\mathrm{Si}, \mathrm{Ge}$ and $\mathrm{C}$ ). The initial configurations, carved from bulk diamond, have all dangling bonds saturated with hydrogens. The structures were relaxed and thermalized for $2-3$ ps at 300 $\mathrm{K}$ and zero pressure, at which point the ionic thermostat was switched off (no electronic thermostat is needed in these simulations). We modeled the adiabatic compression characterizing a shock wave with a linear increase in hydrostatic pressure from 0 to $40 \mathrm{GPa}$ for 1 ps, followed by $0.75 \mathrm{ps}$ at constant pressure and by a linear decrease to $0 \mathrm{GPa}$ in $1 \mathrm{ps}$. The system was finally allowed to evolve at zero pressure for another 810 ps. The structural transformations occurring during the shock are highlighted in Figs. 2 and 3 where the time evolution of the average bond length, quantum volume, and average coordination of the ions are shown for the three nanoparticles. The response of the Si nanoparticle (see Fig. 2) is remarkable: a transition is induced $\sim 0.6$ ps into the compression $(\mathrm{P} \simeq 26-28 \mathrm{GPa})$, at which point the system amorphizes. In this process the second shell of neighbors overlaps and merges with the first, increasing the coordination of the ions (see Fig. (3) from 3.0 - due to the undercoordinated surface atoms - to 5.5. The compressed bonds recover, albeit only on average, the zero-pressure equilibrium length; volume contraction is achieved by a collapse of the coordination shells rather than a shortening of the nearest bonds. The resulting amorphous configuration remains stable after pressure is released, and no recrystallization is detected in the following 8-10 ps. A structural relaxation confirms that the amorphous nanoparticle corresponds to a well-defined local minimum for the potential energy surface; this minimum is $11.15 \mathrm{eV}(0.32 \mathrm{eV}$ per silicon atom $)$ higher than the corresponding initial tetrahedral configuration. These results confirm the existence of an amorphous phase for silicon nanoparticles, as reported in Ref. [5] and also found in the simulations of Refs. [12, 13]. Germanium and carbon at these sizes display instead an elastic response (see Fig. 2) and the original tetrahedral structure is recovered at the end of the simulation. Due 

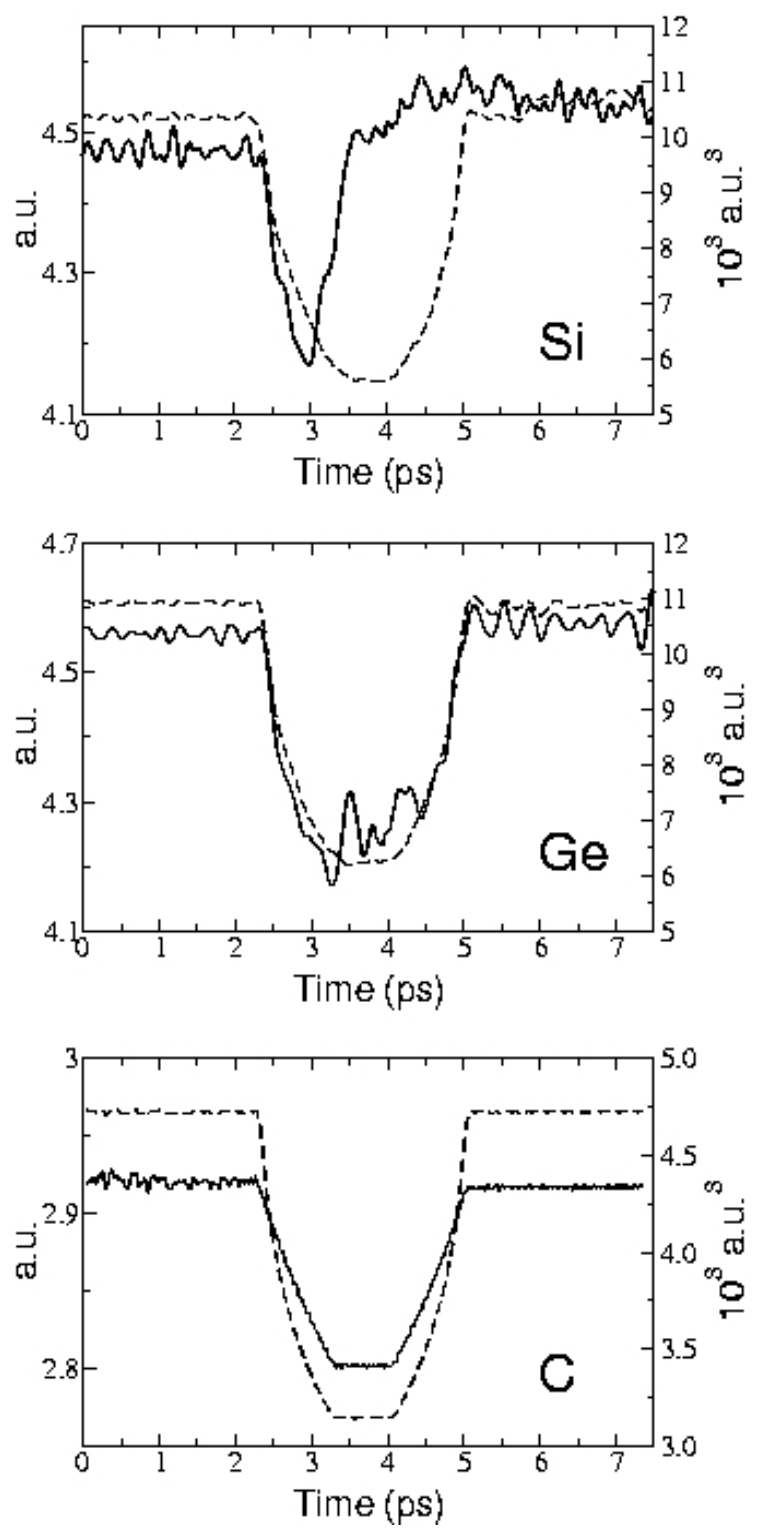

FIG. 2: Time evolution of the average bond length (solid line) and of the quantum volume (dashed line) for the three nanoparticles during the shock wave.

to its very stiff bonds, the carbon nanoparticle maintains its tetrahedral coordination even at peak pressure, while germanium is noticeably softer. In both cases, however, the average coordination of the ions is barely affected (see Fig. 3i) and the first two peaks in the pair-correlation function remain distinct at all times during the shock. The differences observed in the structural behavior are also reflected in the thermodynamics of the compression process. In Fig. 4 we show the mechanical work exerted on the nanoparticles by the compression wave; in our approach this can be straightforwardly determined by $\int P d V$, whereas the fluctuations of an explicit pressurizing medium would hinder the volume changes of the quantum fragment. It is immediately evident that the

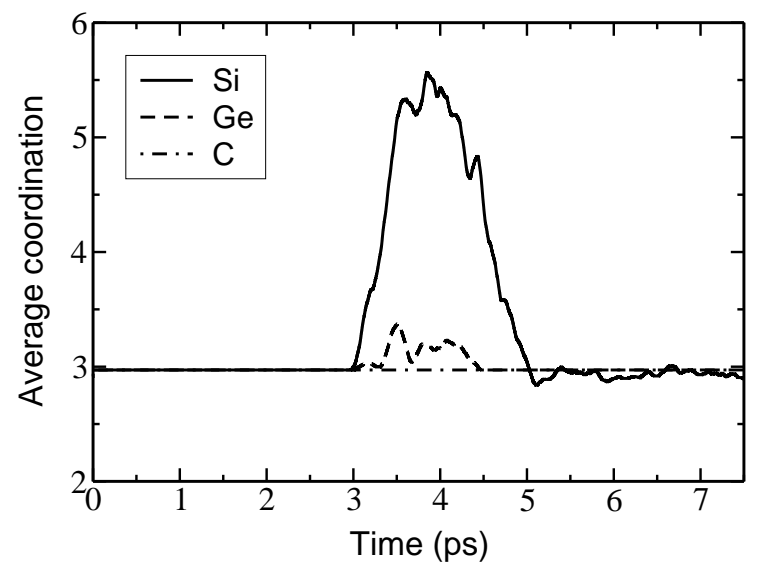

FIG. 3: Time evolution of the average ionic coordination for $\mathrm{Si}$ (solid line), Ge (dashed line) and $\mathrm{C}$ (dot-dashed line) nanoparticles during the shock wave.

Si nanoparticle absorbs a great amount of mechanical energy: a portion of this energy is converted into kinetic energy of the ions, thanks to enhanced anharmonic coupling between acoustic and optical phonons, while a larger part goes into the plastic deformation and transition into the metastable amorphous phase. Both the diamond nanoparticle and (to a lesser extent) the germanium one release instead elastically all the mechanical energy absorbed during the shock and stored in the compression of the covalent bonds.

Kinetic trapping for small Si nanoparticles has been demonstrated in Ref. [5], with an amorphous phase appearing during pressure release below $5 \mathrm{GPa}$. In agreement with Ref. [5], we find an enhancement with respect to the bulk for the pressure transition during the upstroke phase; our nanoparticle falls directly in the amorphous phase, without an intermediate Imma or $\beta$ Sn phase. Pressure-induced amorphization was observed when studying porous silicon films containing diamondstructure nanocrystals under load [24]; amorphization was also found in bulk first-principles simulations 25, 26] in the 11-15 GPa range. The importance of the rate of load release on the structural transformation in $\mathrm{Si}$ and Ge was highlighted in Ref. [27]. We have verified that changing the speed of the shock wave by a factor of $1 / 15$, $1 / 2$, or the initial temperature of the system from $300 \mathrm{~K}$ to $100 \mathrm{~K}$, did not change the amorphization pathway or the transition pressure. We also carefully confirmed that the nanoparticle never becomes liquid.

Finally, we examined the effects that the threshold parameter $\alpha$ defining the quantum volume has on the isoenthalpic dynamics. The silicon simulation was repeated using $\alpha=0.0005$ electrons/(a.u. $)^{3}(\alpha=0.0002$ electrons $/(\text { a.u. })^{3}$ was used for all previous simulations). While at $0 \mathrm{GPa}$ this different choice leads to a volume difference of $\sim 10 \%$, in the course of the compression the two definitions rapidly converge to the same value; the 


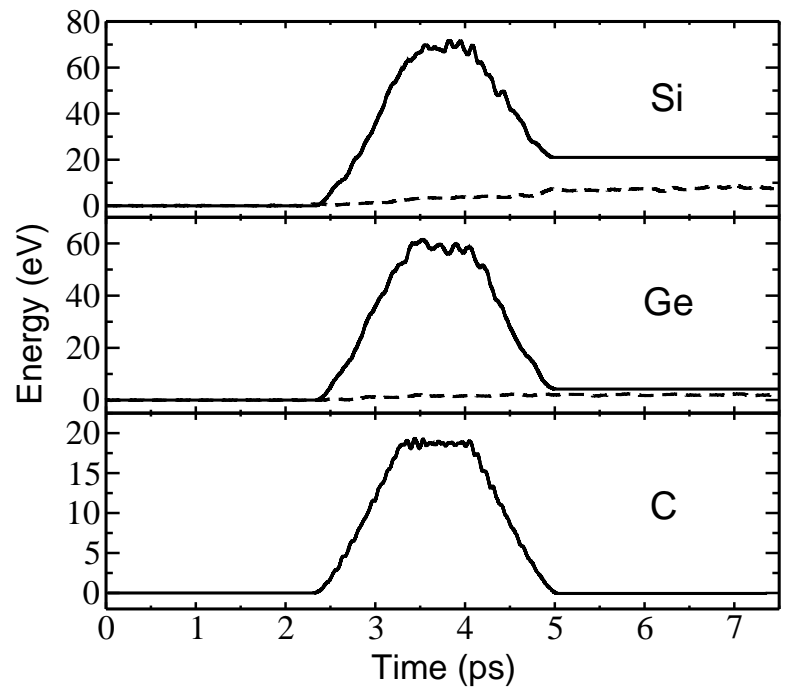

FIG. 4: Work $\int P d V$ done by the external pressure on the nanoparticles during the shock wave (solid line), and instantaneous kinetic energy of the ions (dashed line, zero corresponds to the $300 \mathrm{~K}$ average).

soft "electronic skin" involved when using a lower threshold contracts quickly, and the system then opposes the same resistance to the external load as in the case of a higher threshold. More importantly, the energy absorbed is shown to be the same independently of our choice of threshold; the outer "electronic skin" releases elastically at the end of the run any energy stored at the beginning of the simulation.

In conclusion, we have introduced a novel approach in the study of finite systems under pressure, based on the quantum-mechanical definition of an electronicenthalpy functional. This method is straightforward to implement in static and dynamical first-principles calculations, and opens the study of the phase stability and kinetics of finite quantum systems to extensive firstprinciples simulations. Its accuracy and broad independence on the isosurface threshold have also been established. We have studied the behavior of semiconducting nanoparticles $\left(\mathrm{M}_{35} \mathrm{H}_{36}\right.$, with $\mathrm{M}=\mathrm{Si}, \mathrm{Ge}$, and $\left.\mathrm{C}\right)$ during a hydrostatic shock wave, highlighting the different response of the three materials. Small silicon nanoparticles undergo plastic amorphization at pressures larger than those required to amorphize a bulk system, while carbon nanoparticles (and to a lesser extent germanium ones) elastically recover their original tetrahedral structure 28]. A large amount of mechanical energy is absorbed by silicon nanoparticles as internal energy of their amorphous phase and kinetic energy of the ions. This energy will be released in longer timescales than those of a traveling shock wave, hinting at the possibility of designing a nanostructured impact-absorbing material. It is also envisioned that the transition pressures, decay timescales, and the overall mechanical response could be tuned by an appropriate choice of materials, composi- tion, and sizes. Support from MIT Institute for Soldier Nanotechnologies, grant DAAD19-02-D-0002, is gratefully acknowledged.

[1] S. H. Tolbert and A. P. Alivisatos, Science 265, 373 (1994).

[2] S. H. Tolbert and A. P. Alivisatos, J. Chem. Phys. 102, 4642 (1995).

[3] C. Chen, A. B. Herhold, C. S. Johnson, A. P. Alivisatos, Science 276, 398 (1997).

[4] J. N. Wickham, A. B. Herhold, A. P. Alivisatos, Phys. Rev. Lett. 84. 923 (2000).

[5] S. H. Tolbert, A. B. Herhold, L. E. Brus, A. P. Alivisatos, Phys. Rev. Lett. 76, 4384 (1996).

[6] Z. Wang et al., Appl. Phys. Lett. 83, 3174 (2003).

[7] Z. Wu et al., J. Appl. Phys. 93, 9983 (2003).

[8] H. C Andersen, J. Chem. Phys. 72, 2384 (1980).

[9] M. Parrinello and A. Rahman, Phys. Rev. Lett. 45, 1196 (1980); J. Appl. Phys. 52, 7182 (1981).

[10] R. M. Wentzcovitch, Phys. Rev. B 44, 2358 (1991).

[11] I. Souza and J. L. Martins, Phys. Rev. B 55, 8733 (1997).

[12] R. Martonák, C. Molteni, M. Parrinello, Phys. Rev. Lett. 84, 682 (2000); C. Molteni, R. Martonák, M. Parrinello, J. Chem. Phys. 114, 5358 (2001).

[13] R. Martonák, C. Molteni, M. Parrinello, J. Chem. Phys. 117, 11329 (2002).

[14] E. Curotto, J. Chem. Phys. 114, 10702 (2001).

[15] The smearing width is largely irrelevant in determining the physical response of the system.

[16] R. Car and M. Parrinello, Phys. Rev. Lett. 55, 2471 (1985).

[17] S. Baroni, A. Dal Corso, S. de Gironcoli, P. Giannozzi, C. Cavazzoni, G. Ballabio, S. Scandolo, G. Chiarotti, P. Focher, A. Pasquarello, K. Laasonen, A. Trave, R. Car, N. Marzari, A. Kokalj, http://www.pwscf.org/

[18] J. Tempere, I. F. Silvera, J. T. Devreese, Phys. Rev. Lett. 87, 275301 (2001); M. Rosenblit and J. Jortner, Phys. Rev. Lett. 75, 4079 (1995).

[19] J. L. Fattebert, F. Gygi, J. Comput. Chem. 23, 662 (2002); D. Scherlis et al. (to be published).

[20] H.-P. Cheng, X. Li, R. L. Whetten, and R. S. Berry, Phys. Rev. A 46, 791 (1992).

[21] F. Calvo and J. P. K. Doye, Phys. Rev. B 69, 125414 (2004)

[22] O. H. Nielsen and R. M. Martin, Phys. Rev. Lett. 50, 697 (1983); Phys. Rev. B 32, 3780 (1985); Phys. Rev. B 32, 3792 (1985).

[23] We stress that no fitting procedure has been used to find the agreement among the two curves; the pressure parameter on the graph was the one used in the electronicenthalpy functional.

[24] S. K. Deb et al., Nature 414, 528 (2001).

[25] M. Durandurdu and D. A. Drabold, Phys. Rev. B 67, 212101 (2003).

[26] T. Morishita, Phys. Rev. Lett. 93, 55503 (2004).

[27] A. Mujica et al., Rev. Mod. Phys. 75, 863 (2003).

[28] Larger Ge nanoparticles can also undergo some amorphization. A detailed description of these size effects will be published separately. 